\title{
US stroke guidelines provoke mixed reaction
}

$\mathrm{T}$ he American Heart Association's first-ever guidelines for preventing stroke in women have provoked mixed reaction in the United States and Canada.

Obstetricians are cautioning that some of the recommendations for managing stroke risk in pregnant women may have unknown impacts on mother and child. Meanwhile, stroke experts have lauded the guidelines for raising the visibility of stroke as a women's health issue.

"Many people think of stroke as a disease of aging, and often as a disease of men, when in fact the lifetime risk of stroke is higher for women, and is increased with use of birth control, with pregnancy and with hormone replacement therapies," says Dr. Richard Swartz, director of the stroke program at the University of Toronto in Ontario.

In both Canada and the US, stroke is now more common in women than men. It's also more deadly, with about $60 \%$ of stroke deaths occurring in women, according to the American Heart Association.

Yet, stroke prevention efforts have historically lumped men and women together, says Dr. Gustavo Saposnik, director of the stroke outcomes research unit at St. Michael's Hospital in Toronto. "In the last 10 or 20 years, we were treating them in the same way without realizing women may have different risk factors or overall different risks."

The new guidelines, released Feb. 6 , provide recommendations for managing factors that make women uniquely vulnerable to stroke, including common pregnancy complications, use of birth control pills and hormone replacement therapy, and higher prevalence of migraines and atrial fibrillation. Key recommendations include:

- Women with a history of preeclampsia should be regularly screened and treated for cardiovascular risk factors after pregnancy in recognition of the fact they face a two-fold risk of stroke and four-fold risk of high blood pressure later in life.

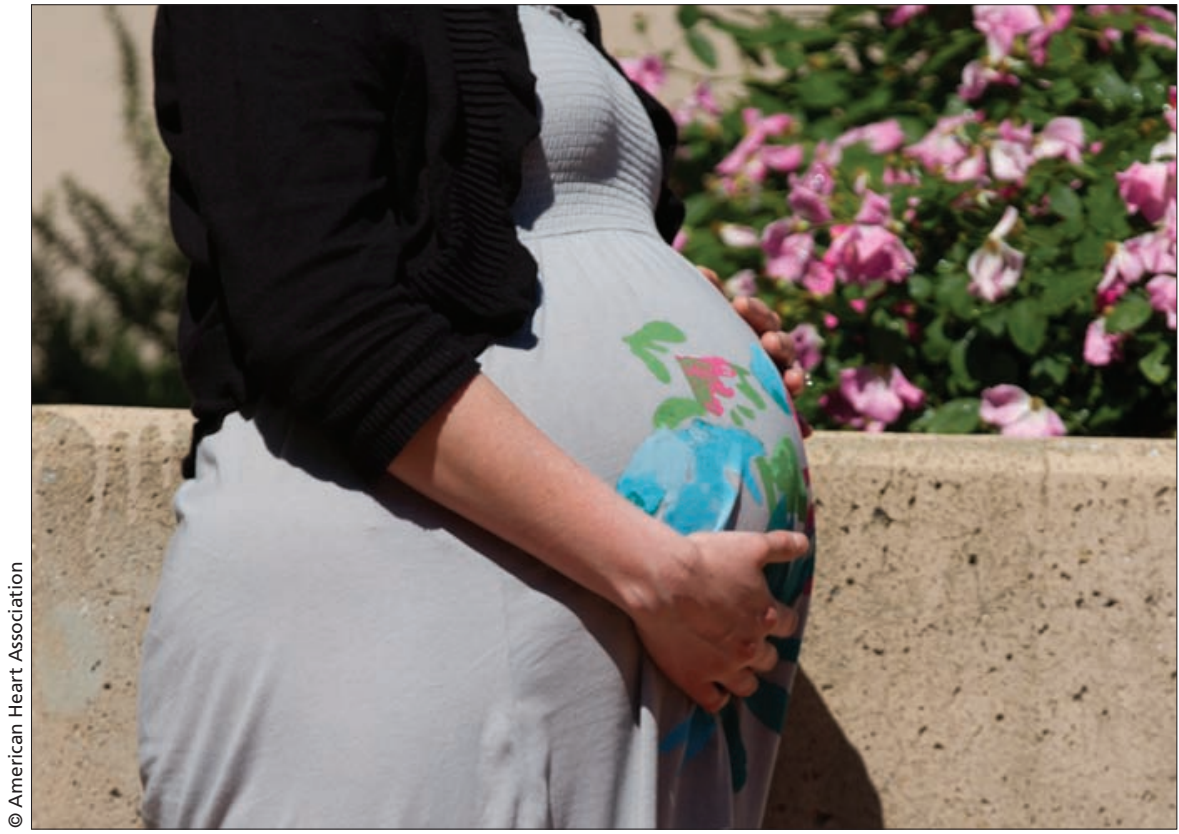

Women with a history of preeclampsia face a two-fold risk of stroke and four-fold risk of high blood pressure later in life, warn new guidelines.

- Women with a history of high blood pressure before pregnancy should be considered for low-dose aspirin and/or calcium supplement therapy to lower preeclampsia risks.

- Pregnant women with moderately high blood pressure (150-159/100$109 \mathrm{mmHg}$ ) should be considered for antihypertensive medications. Those with severely high blood pressure (160/110 $\mathrm{mmHg}$ or above) should be treated.

- Women should be screened for high blood pressure before starting birth control pills.

- Women smokers who have migraines with aura should stop smoking.

- Women over age 75 should be screened for atrial fibrillation, as they're more likely than men to develop the heart rhythm disorder, which increases stroke risk five-fold.

The guidelines set a standard for more targeted prevention, says Dr. Antoine Hakim, chief executive officer and scientific director for the Canadian Stroke Network.
Similar recommendations are already part of more general stroke guidelines in Canada, but the information has never been organized or made searchable by gender, he adds. "It's a good lesson for us and something I'm now committing to do."

Response from obstetricians, however, has been less enthusiastic.

The American Congress of Obstetricians and Gynecologists does not recommend using medications to treat mildly or moderately high blood pressure in pregnant women because the long-term implications are currently unknown, says Dr. James Roberts, who chaired the group's task force on hypertension in pregnancy. The obstetrician group is also more conservative in recommending aspirin treatment.

Calcium supplementation wasn't even discussed, adds Roberts, because patients with low enough calcium intakes to receive benefit from supplements are rare.

According to Dr. Graeme Smith, head of obstetrics and gynecology at Queen's University in Kingston, 
Ontario, "part of the problem is that there's no absolute in how we should be managing these people."

Smith says it's unlikely the treatment recommendations will have "any impact" where they differ in specifics from obstetricians' current practices, particularly as overtreating can be risky. "There's a potential you may drop her blood pressure enough to have an adverse impact on [placental blood circulation] and fetal growth."

Taken as a whole, however, the guidelines represent an exciting shift away from traditional thinking about pregnancy as an isolated event in a woman's life, he adds. Smith's clinic is one of only two in North America that continue to follow patients after delivery.
"The take-home message is that these women are at greater risk for future health complications and there should be some plan in place for follow-up," he says. "We've known this data for 10 to 15 years, but it's only now being translated into clinical practice." — Lauren Vogel, CMAJ

CMAJ 2014. DOI:10.1503/cmaj.109-4732 